Erfahrungsaustausch beim bad

\title{
Entbürokratisierung der Pflegedokumentation
}

— Die Entbürokratisierung der Pflegedokumentation ist ein beherrschendes Thema der Branche. Beim Bundesverband Ambulante Dienste und stationäre Einrichtungen (bad) nahm Elisabeth Beikirch - im Auftrag des BMG zuständig für das neue Dokumentationsmodell - Stellung zur praktischen Umsetzung des Modells. Der bad hatte Mitglieder, die bereits die Umstellung vorgenommen haben, nach Essen eingeladen, um mit ihnen Praxiserfahrungen zu diskutieren. Begrüßt wurde der Hinweis von Beikirch, dass sich auch Einrichtungen der Tagespflege auf der Internetseite des Projektbüros registrieren lassen können. „Da viele ambulante Dienste gleichzeitig auch eine Tagespflege betreiben, macht es keinen Sinn, dass ambulant nach dem Strukturmodell gearbeitet werden kann, die Dokumentation in der Tagespflege aber die herkömmlichen Strukturen aufweist", sagte Andreas Kern, Bundesvorsitzender des bad. „Der Erfahrungsaustausch hat die Vorteile der neuen Dokumentation für die Einrichtungen, die Pflegekräfte, aber auch für die gepflegten Personen nochmal deutlich gemacht", resümierte Kern. Die Erfahrungen zeigen, dass niemandem empfohlen werden kann, die Umstellung gänzlich ohne Hilfe zu bewerkstelligen. Hier sieht Andrea Kapp, Bundesgeschäftsführerin des bad, die Verbände in der Pflicht: „Der bad wird sicherstellen, dass die flächendeckende Umsetzung des Projekts, von dem wir inhaltlich überzeugt sind, weiter vorangetrieben wird."

www.ein-step.de

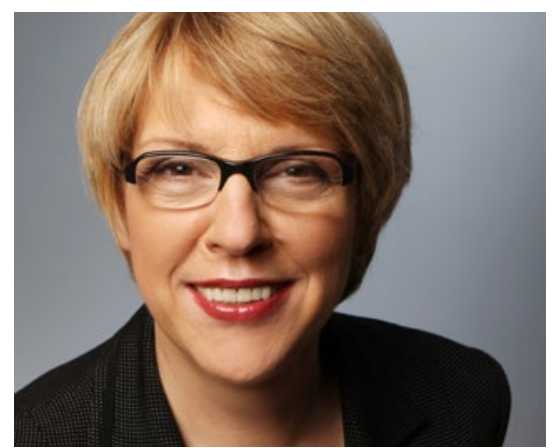

KTQ Manual

\section{Mehr Sicherheit}

— Die überarbeitete, vollständige Weiterentwicklung des KTQ Manuals 2015 liegt jetzt vor. Gerade für den Bereich der Rezertifizierung ist diese Überarbeitung wichtig. Durch die prominenten Gesellschafter - die Deutsche Ärzteschaft, die Krankenhausgesellschaft, der Deutsche Pflegerat und die gesetzlichen Krankenkassen - nimmt das KTQ-Zertifizierungsverfahren eine Spitzenposition innerhalb der Zertifizierer in Deutschland ein. Im Fokus der Weiterentwicklung stand das Thema Risikomanagement und damit die Berücksichtigung der im Jahr 2014 aktualisierten Qualitätsmanagement-Richtlinie Krankenhäuser (KQM$\mathrm{RL})$ des Gemeinsamen Bundesausschuss. Der neue KTQ-Katalog soll die Mitarbeiter in besonderer Weise für die Themen wie Sicherheitskultur sensibilisieren.

www.ktq.de

\section{Jobmotor Nummer eins in Deutschland}

- Die Branche Pflege und Soziales verzeichnete im Oktober 2015 laut Arbeitsmarktbericht Dezember 2015 der Bundesagentur für Arbeit mit plus 93.000 sozialversicherungspflichtigen Beschäftigten den absolut größten Zuwachs aller Branchen gegenüber dem Vorjahr. Sie lag damit vor dem Handel (+83.000), sonstige wirtschaftliche Dienstleistungen $(+75.000)$ sowie qualifizierte Unternehmensdienstleistungen (+69.000).

Insgesamt wuchs die sozialversicherungspflichtige Beschäftigung in diesem Zeitraum um 713.000 Stellen. „Jede siebte neue sozialversicherungspflichtige Beschäftigung ist im letzten Jahr im Bereich Pflege und Soziales entstanden. Damit ist die Branche Jobmotor Nummer eins in Deutschland", erklärte Rainer Brüderle, Präsident des bpa Arbeitgeberverbandes. Und weiter: „In der Pflege entstehen die sichersten Arbeitsplätze der nächsten Jahr- zehnte. Es steht also besser um die Berufsaussichten im Pflege- und Sozialbereich als es von vielen Seiten suggeriert wird - immer mehr Jobs entstehen hier und immer mehr Menschen entscheiden sich für genau diesen Beruf. Die Zahlen machen deutlich, dass wir uns mehr Gedanken über den Wert der Pflege in und für unsere Gesellschaft machen müssen. Es läuft doch etwas schief, wenn Leistungen der Pflege deutlich schlechter vergütet werden als andere vergleichbare Dienstleistungen. So ist jeder bereit, für die Anfahrt eines Handwerkers 35 Euro zu bezahlen, für die Anfahrt eines Pflegedienstes werden aber oft nur 3,70 Euro vergütet. Wer also in Sonntagsreden über die Pflege spricht, sollte sich auch von Montag bis Freitag um eine entsprechende und wertschätzende Vergütung kümmern", so Brüderle.

www.bpa.de
Alles richtig?

Die richtigen Antworten der PflegeKollegs lauten:

Sofortmaßnahmen:

$1 b, 2 b, 3 c, 4 c, 5 b, 6 a, 7 a, 8 b, 9 a, 10 a$

Sepsis:

$1 a, 2 a, 3 c, 4 a, 5 b, 6 a, 7 b, 8 a, 9 b, 10 c$

Vorschau!

Die PflegeKollegs im März:

Geriatrie: Die Alten kommen Mobilisation - Ethik - Neue Konzepte

Parasiten auf Haut \& Haar Krätze - Läuse - Milben 